\title{
Categorical color coding by pigeons
}

\author{
THOMAS R. ZENTALL and CHARLES A. EDWARDS \\ University of Kentucky, Lexington, Kentucky
}

\begin{abstract}
Pigeons were trained on two independent tasks. One involved red and yellow hues, the other involved blue and green hues. For half of the birds, the two tasks were the same (i.e., both tasks were either matching-to-sample, or oddity-from-sample). For the remaining birds, the two tasks were different (i.e., one task was matching-to-sample; the other task was oddity-from-sample). Following acquisition, the pigeons were exposed to test trials on which either the correct or the incorrect comparison hue was replaced with one of the hues from the other task. On yellow-sample trials and on green-sample trials, the pigeons performed as if they had a common code for yellow and green. When there was one comparison available that was appropriate to the "yellow/green" code, performance remained high; but when either both comparisons or neither comparison was appropriate to the "yellow/green" code, performance dropped. The pigeons also tended to code red samples as green and to code blue samples as yellow. The results indicate that pigeons can categorically code colors under conditions that rule out a failure to discriminate among the colors.
\end{abstract}

A code can be described as the transformation of a stimulus, such that it is represented by more than or less than the absolute physical properties of the stimulus.

In some cases, the code can be relational, in the sense that it involves those aspects of the stimulus that distinguish it from other relevant stimuli. Thus, the code associated with a small white circle could involve size if the stimulus was contrasted with a large white circle, brightness if the stimulus was contrasted with a small black circle, or shape if the stimulus was contrasted with a small white square. The notion of dimensional coding was introduced by Lawrence (1963), and it formed the basis for Sutherland and Mackintosh's (1971) attentional theory of animal discrimination learning.

In other cases, the code can be an arbitrary overt response that intervenes between a presented stimulus and a required response (e.g., in a memory task). To be more specific, when pigeons are exposed to a delayed, conditional-discrimination task, they develop idiosyncratic, stimulus-specific, delay behavior that can serve as a salient representation of the conditional stimulus, at the time of choice (Blough, 1959; Zentall, Hogan, Howard, \& Moore, 1978).

More generally, however, the term "coding" is used to indicate any means of categorizing, grouping, or labeling stimuli. The term is particularly useful under conditions in which simple stimulus generalization (i.e., the monotonic increase in response strength as a function of stimulus similarity) cannot provide a complete account of the data.

This research was supported by Grant MH35378 from the National Institute of Mental Health to the first author. We thank an anonymous reviewer for comments on an earlier version of this manuscript. Charles Edwards is now at the Department of Psychology, Dalhousie University, Halifax, N.S., Canada B3H 4JI. Send reprint requests to T. R. Zentall, Department of Psychology, University of Kentucky, Lexington, KY 40506.
Evidence for the categorical coding of stimulus hues has been reported by Cumming and his associates (Cumming, Berryman, \& Cohen, 1965; Berryman, Cumming, Cohen, \& Johnson, 1965). They have argued that during conditional discrimination learning pigeons develop codes for each of the conditional (or sample) stimuli. That these codes are categorical in nature is suggested by the observation that pigeons will use an existing code when confronted with a new sample stimulus. For example, after pigeons had learned a matching-to-sample task that involved a red sample on some trials, a green sample on other trials, and a blue sample on still other trials, novel yellow stimuli were introduced in place of the blue. The transfer data suggested that the pigeons treated the novel yellow sample (but not the novel yellow comparison) "as if it were red" (Cumming et al., 1965). Thus, on trials with a novel yellow sample and a choice between a red and a yellow comparison stimulus, the pigeons selected the "incorrect" red comparison stimulus $83 \%$ of the time. The pigeons selected the red alternative presumably because, in the presence of a sample stimulus "coded as red," a response to red had been reinforced. However, on trials with a novel yellow sample and a choice between a green and a yellow comparison stimulus, the pigeons selected the "incorrect" green comparison stimulus only $60 \%$ of the time. They did so because in the presence of a sample stimulus "coded as red" the pigeons were relatively indifferent to the yellow and green alternatives.

The data appear to support the coding hypothesis, but a more parsimonious explanation can be given for the general pattern of results obtained when pigeons are given a choice between a novel and a familiar comparison stimulus. If, independent of the sample, one assumes that a pigeon will select a familiar stimulus to which responses have been often reinforced, rather than a novel stimulus not associated with a prior history of reinforcement, then 
one would expect poor performance when the novel stimulus was defined as correct and good performance when the familiar stimulus was defined as correct (see Zentall \& Hogan, 1974). In fact, in the experiment reported by Cumming et al. (1965), the pigeons performed above $90 \%$ correct on novel trials that involved red or green samples (i.e., the correct comparison stimulus was either red or green, and the incorrect comparison stimulus was yellow). Some support for the coding hypothesis remains, however, because the novel-stimulus account of the transfer data predicts no difference in level of performance between yellow-sample trials with a red incorrect comparison (which was $17 \%$ correct) and yellow-sample trials with a green incorrect comparison (which was $40 \%$ correct).

Similar performance differences were reported by Berryman et al. (1965) on novel yellow-sample trials following oddity-from-sample training (the correct comparison is the one that does not match the sample). During training, the birds had learned, for example, to peck green when the sample was red. Thus, on novel yellowsample trials when the correct comparison was green, performance level was high ( $96 \%$ correct) presumably because the yellow sample was coded as red. When the correct comparison was red, however, performance level was low ( $54 \%$ correct) because during training the birds had not learned to peck red in the presence of a sample coded as red.

In the procedure used by Cumming and his colleagues, if coding occurred, each sample used during training had to be coded differently for task acquisition to occur. There is some evidence, however, that if the procedure used is one that allows for different samples to be given a common code, common coding can occur (Zentall, Edwards, Moore, \& Hogan, 1981, Experiment 1). Zentall et al. (1981) trained pigeons with two independent matching or oddity tasks. Trials from one task were interspersed with trials from the other task. One task involved red and green hues; the other task involved yellow and blue hues. In order to assess what had been learned during training, one of the two hues from one task replaced either the correct or the incorrect alternative on the other task. The major finding was that when the exchange was between red and yellow or blue and green, performance remained high, but when the exchange was between red and blue or yellow and green, performance dropped to near chance level. In this two-task procedure there is no need for the pigeons to code these hue pairs differentially, because during training red and yellow (and blue and green) are never presented together on the same trial. A pigeon could thus perform well during training by learning two "categorical coding rules" (e.g., for a matching bird: if the sample is "red/yellow," peck the red or the yellow comparison, and if the sample is "green/blue," peck the green or the blue comparison). Thus, on test trials that involved a choice between red and yellow comparisons, or between green and blue comparisons, performance was poor because the categorical rule learned during training was ambiguous (i.e., it no longer provided sufficient information). On trials that involved a choice between red and blue comparisons, or between yellow and green comparisons, however, the level of performance remained high because the categorical rule learned during training could be unambiguously applied (i.e., there was one and only one response choice appropriate given the rule learned).

One can, of course, propose an alternative explanation for the above findings. It is possible that since the pigeons had not been explicitly trained to discriminate yellow from red, and blue from green, they "confused" these color pairs and responded to them not because they were coded the same, but because they could not tell them apart (i.e., there was a failure to discriminate stimulus differences). Given the large nominal difference in wavelength between the red/yellow and blue/green hue pairs, it seems unlikely that the members of the pairs were perceived as the same. Prokasy and Hall (1963) have argued, however, that in the absence of explicit discrimination training, considerable failure of discrimination can be found, even among potentially highly discriminable stimuli.

The main purpose of the present experiment was to rule out discrimination failure as an explanation for the data reported by Zentall et al. (1981, Experiment 1). The present experiment involved four training groups. Each group was trained on two tasks (interspersed trials). One of the two tasks involved the hues red and yellow, the other task involved the hues green and blue. The hues used in the present experiment were the same as those used by Zentall et al. (1981). The hue pairings were altered, however, to assess the generality of the earlier finding. For Group MM, both tasks were matching-to-sample. For Group OO, both tasks were oddity-from-sample. For Group MO, the red-yellow task was matching-to-sample and the green-blue task was oddity-from-sample. For Group OM, the red-yellow task was oddity-from-sample and the green-blue task was matching-to-sample.

Given the relative spectral proximity of yellow and green, it was expected that the pigeons would code yellow as green and green as yellow. Similarly, if the pigeon's psychological color space is circular (Schneider, 1972), as is the psychological color space for humans (Boynton, 1972, pp. 346-347), one would expect that the pigeons would code red as blue and code blue as red. Assuming that these codes are learned, coding rules for the four groups of birds can be identified (see Table 1). These coding rules can then be applied to the specific trial types used during testing to make predictions about performance level. The training and test trials used in the present study are presented in Table 2.

If, for Groups MM and OO, poor performance were to be found on trials with certain hue pairs (e.g., yellow/green) but not with others (e.g., yellow/blue), then performance on the same trials by the two mixed-task groups (MO and OM) would provide a critical test of the categorical coding hypothesis. According to the failure- 
Table 1

Possible Coding Rules for the Four Groups of Pigeons

\begin{tabular}{|c|c|c|}
\hline Group & If the sample is & $\begin{array}{l}\text { Then peck the com- } \\
\text { parison that is }\end{array}$ \\
\hline MM & $\begin{array}{l}\text { yellow/green } \\
\text { red/blue }\end{array}$ & $\begin{array}{l}\text { yellow or green } \\
\text { red or blue }\end{array}$ \\
\hline OO & $\begin{array}{l}\text { yellow/green } \\
\text { red/blue }\end{array}$ & $\begin{array}{l}\text { red or blue } \\
\text { yellow or green }\end{array}$ \\
\hline MO & $\begin{array}{l}\text { yellow/green } \\
\text { red/blue }\end{array}$ & $\begin{array}{l}\text { yellow or blue } \\
\text { red or green }\end{array}$ \\
\hline OM & $\begin{array}{l}\text { yellow/green } \\
\text { red/blue }\end{array}$ & $\begin{array}{l}\text { red or green } \\
\text { yellow or blue }\end{array}$ \\
\hline
\end{tabular}

Table 2

Trial Types on Which All Pigeons Were Trained and Tested

Test

\begin{tabular}{ccccc} 
Training & \multicolumn{2}{c}{$\begin{array}{c}\text { Matching New Incorrect, } \\
\text { Oddity New Correct }\end{array}$} & \multicolumn{2}{c}{$\begin{array}{c}\text { Matching New Correct, } \\
\text { Oddity New Incorrect }\end{array}$} \\
\hline Y(YR) & Y(YG) & Y(YB) & Y(GR) & Y(BR) \\
G(BG) & G(YG) & G(RG) & G(BY) & G(BR) \\
R(YR) & R(GR) & R(BR) & R(YG) & R(YB) \\
B(BG) & B(BY) & B(BR) & B(YG) & B(RG) \\
\hline
\end{tabular}

Note-First letter represents sample color. Letters in parentheses represent comparison colors. $Y=$ yellow, $R=$ red, $G=$ green, $B=$ blue.

of-discrimination hypothesis, if poor performance by Groups MM and OO on, for example, yellow-green test trials (e.g., yellow sample with yellow and green comparisons) was due to a failure to discriminate those colors, then similarly poor performance should be obtained for the two mixed-task groups on those trials. According to the categorical coding hypothesis, however, performance by the mixed-task groups on yellow-green trials should be high because the rule learned by a Group MO bird is, "If the sample is yellow/green then peck yellow or blue." Thus, given a yellow sample (or a green sample) and a choice between yellow and green comparisons, the bird should choose yellow (the correct matching response), because green had never been correct in the presence of a "yellow/green" sample stimulus. On the other hand, given a yellow sample (or a green sample) and a choice between yellow and blue comparisons, the bird should be in conflict and performance should suffer because both yellow and blue had been correct in the presence of a "yellow/green" sample stimulus.

\section{METHOD}

\section{Subjects}

Sixteen experimentally naive female White Carneaux pigeons, approximately 1 year old, served as subjects. The birds were deprived to and maintained at $75 \%-80 \%$ of free-feeding weight. Water and grit were available in their home cages.

\section{Apparatus}

A standard pigeon operant chamber (inside dimensions: $35 \mathrm{~cm}$ high, $30 \mathrm{~cm}$ wide, and $35 \mathrm{~cm}$ across the intelligence panel) was used for training and testing. Three rectangular pecking keys $(3.2 \times 2.5 \mathrm{~cm})$ were centrally mounted side by side on the intelligence panel. The bottom edge of the keys was $16.0 \mathrm{~cm}$ above the chamber floor and $8.0 \mathrm{~cm}$ above the top edge of a centrally positioned grain feeder opening. Behind each pecking key was an in-line projector that could illuminate the keys with a red, green, yellow, or blue field (Kodak Wratten filters, Nos. 26, 60, $9,38 \mathrm{~A}$, respectively). The hues were matched for brightness (Blough, 1957) with Kodak neutral density filters. Experimental sessions were controlled by solid-state equipment located in an adjoining room. Sound masking was provided by white noise and a blower fan.

\section{Procedure}

All birds were magazine trained and trained to peck a lit response key. The hue of the key to which each bird was trained to peck and its position (left or right) were counterbalanced. On the next 2 days, each bird was trained to respond to a single lit key that changed position and hue randomly following each of 48 reinforcements.

Training. On the next day, each bird was assigned randomly to one of four training groups: matching-matching (MM), oddity-oddity $(O O)$, matching-oddity (MO), and oddity-matching (OM). Group MM was trained with a red-yellow matching task and a blue-green matching task. Group $\mathrm{OO}$ was trained with a red-yellow oddity task and a blue-green oddity task. Group MO was trained with a red-yellow matching task and a blue-green oddity task. Group OM was trained with a red-yellow oddity task and a blue-green matching task.

For all birds, whenever the sample was red or yellow, the comparison stimuli were red and yellow, and whenever the samples were blue or green, the comparison stimuli were blue and green. At the start of each trial the center key (sample) was lit. Five pecks to the sample illuminated the side keys (comparisons). The sample remained on. A single peck to either comparison terminated the trial and initiated a 5-sec intertrial interval. Correct comparison-key responses were reinforced with 2.5 -sec access to Purina pigeon grains. Hue of the sample and position of the correct comparison stimulus were counterbalanced over the 96 trials in each session. Stimulus presentations for the matching and oddity tasks were identical; only the position of the correct response differed. The training phase consisted of 14 sessions.

Test. Each of the four test sessions consisted of the presentation of control trials (32) and experimental trials (64). Control trials were the same as training trials. These were included to assess general performance disruption due to the inclusion of experimental trials. Experimental trials consisted of four types. Given a particular sample hue. either the correct or the incorrect comparison stimulus was replaced by either one or the other stimulus from the other task. Within a test session for a particular bird, one hue replaced the correct comparison and the other hue replaced the incorrect comparison. This assignment was counterbalanced over the four birds in each group and also over the four test sessions in an ABBA order.

\section{RESULTS}

\section{Training}

The four groups did not acquire their training tasks at the same rate. The two groups exposed to only matching or only oddity tasks (Groups MM and OO) learned faster than the two groups exposed to both tasks (Groups MO and $\mathrm{OM}$ ). The acquisition data are presented in Figure 1. A one-way analysis of variance performed on the training data, pooled over (as many as) the first nine training sessions, indicated that the difference in performance between the common-task groups (MM and OO pooled) and the mixed-task groups (MO and OM pooled) was significant $[F(1,14)=5.25]$. Significance is defined here and in all further analyses by attainment of the .05 level of confidence. Acquisition differences were also indicated by a significant difference between the common-task and mixed-task groups in sessions to a criterion of $75 \%$ correct $[\mathrm{F}(1,14)=7.30]$. 


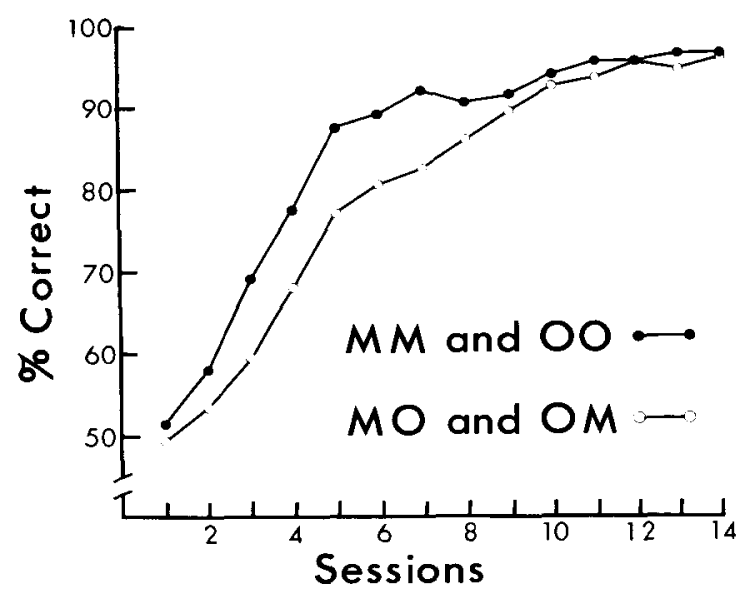

Figure 1. Acquisition functions for pigeons that were trained on the same task (both matching or both oddity, MM and $O O$ ) and pigeons that were trained on different tasks (one matching and one oddity, MO and $\mathrm{OM}$ ).

\section{Test}

The test data that involved yellow and green samples differed considerably from the data that involved red and blue samples in terms of the extent and kind of categorical coding. For this reason, test trials involving each of the different hue samples were analyzed separately. Performance on control trials was uniformly high, so control data were not analyzed further.

According to the coding hypothesis, if yellow and green samples were given the same categorical code, then performance would not be disrupted on test trials when the training rule associated with the sample hue was still appropriate (e.g., for the MM group, the sample rule learned during training, if "yellow/green" respond to yellow or green, would still be appropriate to both a yellowsample/green-red-comparison trial and to a yellowsample/yellow-blue-comparison trial). Test-trial performance would be disrupted, however, when the training rule was no longer appropriate (e.g., for the MM group, the sample rule learned during training was inappropriate to both a yellow-sample/blue-red-comparison trial and to a yellow-sample/yellow-green-comparison trial).

A repeated measures analysis of variance was performed on the data for each sample hue. The variables analyzed were group (MM, OO, MO, and OM), appropriateness of the training rule (appropriate or inappropriate), and whether the replaced stimulus had been the correct or incorrect comparison (new correct or new incorrect).

Yellow sample trials. Performance on "yellow/green"rule-appropriate trials $(92.8 \%$ correct) was significantly better than performance on "yellow/green"-ruleinappropriate trials $(61.9 \%$ correct $)[F(1,12)=156.68]$. Performance by Groups MO and OM on the critical trial types (viz, yellow-sample/yellow-green-comparison trials) showed a similar effect. For Group MO, performance on rule-appropriate, yellow-sample/yellow-green-comparison trials $(98.4 \%$ correct) was considerably better than performance on rule-inappropriate yellow-sample/yellowblue-comparison trials $(62.5 \%$ correct $)$. Similarly, for Group OM, performance on rule-appropriate, yellowsample/yellow-green-comparison trials ( $90.6 \%$ correct) was substantially better than performance on ruleinappropriate, yellow-sample/yellow-blue-comparison trials $(43.8 \%$ correct).

In addition to the large main effect of appropriateness, there was a significant, though much smaller, group effect $[F(3,12)=5.09]$ and a significant group $\times$ appropriateness interaction $[F(3,12)=4.29]$. The latter effect was largely produced by better performance on ruleinappropriate trials by Group OO (76.6\% correct) than by the other three groups $(60.2 \%, 57.8 \%$, and $53.1 \%$ correct for Groups MM, MO, and OM, respectively). The test data are presented in Table 3 separately for each sample.

Green sample trials. Performance on "yellow/green"rule-appropriate trials $(92.4 \%$ correct $)$ was significantly better than performance on "yellow/green"-ruleinappropriate trials $(61.2 \%$ correct $)[\mathrm{F}(1,12)=143.37]$. Performance by Groups MO and OM on the critical trial types (viz, green-sample/yellow-green-comparison trials) showed a similar effect. For Group MO performance on the rule-appropriate green-sample/yellow-greencomparison trials $(95.3 \%$ correct) was considerably better than performance on rule-inappropriate greensample/red-green-comparison trials $(51.6 \%$ correct). Similarly, for Group OM, performance on the ruleappropriate green-sample/yellow-green-comparison trials (90.6\% correct) was considerably better than performance on rule-inappropriate green-sample/green-red-comparison trials $(57.8 \%$ correct $)$. None of the other effects was reliable.

Red sample trials. Performance on "red-blue"-ruleappropriate trials $(73.0 \%$ correct) was significantly worse than performance on "red/blue"-rule-inappropriate trials $(84.6 \%$ correct) $[\mathrm{F}(1,12)=7.43]$. Thus, red samples were coded as green rather than blue. Critical red-sample trials were not examined because performance on these trials was to serve to distinguish between red/blue coding and a failure to discriminate, and no evidence for red/blue coding was found.

A significant new-correct/new-incorrect effect was also found $[F(1,12)=13.99]$. Performance on trials on which the incorrect comparison was replaced $(85.5 \%$ correct) was better than performance on trials on which the correct comparison was replaced $(72.1 \%$ correct). Two significant interactions were also found: groups $\times$ appropriateness $[F(3,12)=8.25]$ and groups $\times$ new correct/ new incorrect $[F(3,12)=3.70]$.

The groups $\times$ appropriateness interaction is attributable to the fact that although the mixed-task groups (Groups $\mathrm{MO}$ and $\mathrm{OM}$ ) performed better on ruleinappropriate trials $\mathbf{9 3 . 0 \%}$ and $\mathbf{8 9 . 8 \%}$ correct, respectively) than on rule-appropriate trials $(54.7 \%$ and $64.1 \%$ correct, respectively), the common task groups 
Table 3

Mean Performance (Percentage Correct) for Each Group on Each Trial Type

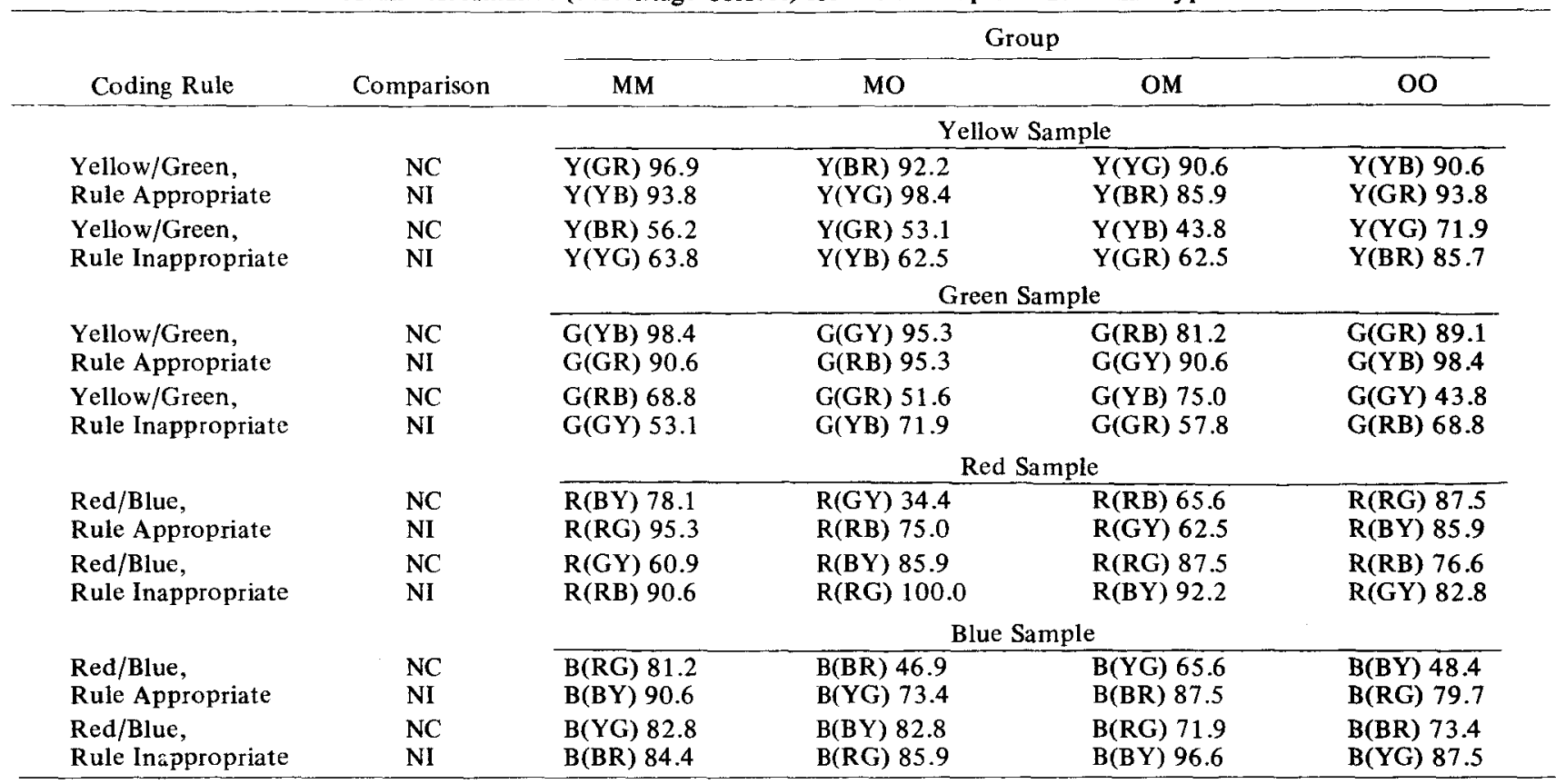

Note $-N C=$ new correct $; N I=$ new incorrect.

(Groups MM and $\mathrm{OO}$ ) actually performed better on rule-appropriate trials (both $86.7 \%$ correct) than on rule-inappropriate trials $\mathbf{( 7 5 . 8 \%}$ and $79.7 \%$ correct, respectively).

The groups $\times$ new-correct/new-incorrect interaction is attributable to the fact that performance on new incorrect trials was better than performance on new correct trials primarily for Groups MM and MO (the two groups for which the red sample was matched). Although the mean difference in performance between new-incorrect and new-correct trials was $25.4 \%$ correct for the two groups that were trained on a red matching task, the difference in performance was only $1.5 \%$ correct for the two groups that were trained on a red oddity task.

Blue sample trials. Performance on "red/blue"'-ruleappropriate trials $(71.7 \%$ correct) was significantly worse than performance on "red/blue"-rule-inappropriate trials $(83.2 \%$ correct $)[F(1,12)=6.23]$. Thus, blue samples were coded as yellow rather than red.

As with red-sample trials, a significant new-correct/ new-incorrect effect was found with blue-sample trials $[F(1,12)=9.62]$. Performance on trials on which the incorrect comparison was replaced $(85.8 \%$ correct) was better than performance on trials on which the correct comparison was replaced $(69.1 \%$ correct).

For the blue-sample trials, a significant group effect was also found $[F(3,12)=3.91]$. Both groups that were trained on a blue matching task (Groups MM and OM) performed better $(84.8 \%$ and $80.5 \%$ correct, respectively) than the groups that were trained on a blue oddity task (Groups MO and OO, both $72.3 \%$ correct).
A significant appropriateness $\times$ new-correct/newincorrect interaction was also found for the blue-sample trials $[F(1,12)=5.47]$. The interaction can be attributed to the fact that the pigeons did especially poorly when "red/blue"-rule-appropriate trials involved replacement of the correct comparison $(60.6 \%$ correct) as compared with performance under the other three conditions $(77.5 \%$ correct on new-correct/rule-inappropriate trials, $82.5 \%$ correct on new-incorrect/rule-appropriate trials, and $88.8 \%$ correct on new-incorrect/rule-inappropriate trials).

\section{DISCUSSION}

The test results indicate that yellow and green samples were commonly coded as "yellow/green." The results also indicate that red samples were coded as green and blue samples were coded as yellow. The critical contrasts for Groups MO and OM, to distinguish between common coding and generalization or confusion between green and yellow, were comparisons of performance (1) on yellowsample/yellow-green-comparison trials and yellowsample/yellow-blue-comparison trials, and (2) on green-sample/green-yellow-comparison trials and greensample/green-red-comparison trials. The results showed clearly that the pigeons were categorically coding yellow and green and were not confusing them. If one prefers a less cognitive description of the data, one might say that the pigeons were able to discriminate almost perfectly between yellow and green when these colors were presented simultaneously as comparison stimuli, but at the same time showed almost complete generalization between yellow 
and green when these same colors were presented successively as sample stimuli. However, such an "objective" description of the data does little to clarify the results and paradoxically is less parsimonious than an explanation based on categorical coding.

Why did pigeons in the present study use the categorical code "yellow/green" whereas in previous research (Zentall et al., 1981), pigeons used the categorical codes "red/ yellow" and "green/blue"? Keep in mind that the same values were used in both experiments; only the pairing of those hues differed. It is possible that the pigeon is relatively flexible in its use of categorical codes and that, within limits, it can define a category so as to facilitate task acquisition. It may be easier for the pigeon to learn the rule "if the sample is red/yellow, peck the red or the yellow comparison" than it is to learn "if the sample is red, peck the red comparison" and "if the sample is yellow, peck the yellow comparison." However, if the use of a $\mathrm{red} / \mathrm{yellow}$ categorical code is precluded by the task, as it is in the present experiment (red and yellow hues needed to be discriminated for task acquisition to occur), then other acquisition-facilitating categories (e.g., yellow/ green) may be used.

Alternatively, it is possible that pigeons have natural (species specific) categories for hues that are relatively inflexible. Natural color categories have been reported for humans (Berlin \& Kay, 1969; Heider, 1972), as demonstrated by the fact that if people from different cultures are presented with an array of different colors they will tend to select the same exemplars as the "focal" or "prototype" of the color names that their culture uses (e.g., given a large number of spectrally different "red" hues, cultures show remarkable agreement on what is the best example of the color that we call red). Some support for the notion of natural color categories for pigeons comes from research by Wright and Cumming (1971). They found that all hues below $535 \mathrm{~nm}$ (blue through green) were treated as if they came from the same category, as were all hues between 535 and $600 \mathrm{~nm}$ (green through yellow) and all hues above $600 \mathrm{~nm}$ (yellow through red). Since the yellow and green Kodak Wratten filters used in the present study had peak transmission values at about 582 and $535 \mathrm{~nm}$, respectively, they fell near the boundaries of Wright and Cumming's yellow/green category. The fact that Zentall et al. (1981) found evidence for categorical red/yellow coding and blue/green coding is also consistent with Wright and Cumming's data. Given that the yellow and green filters used in the present study fell close to Wright and Cumming's color name boundaries, pigeons may sometimes categorically code yellow and green, but at other times categorically code yellow and red, and green and blue; which they do will depend on which of the hues are paired during discrimination training. An example from human color categorization may help clarify this point. If a human subject were presented the color turquoise, a color that falls near the bluegreen boundary, the category to which it was assigned might depend on whether it was contrasted with green (in which case it might be categorized as blue) or with blue (in which case it might be categorized as green).

But even if there are natural color categories for the pigeon, the fact that the same spectral values can be categorized differently, depending on which stimuli are paired during training, suggests that there is some flexibility in the boundaries of the color categories.

The red-sample and blue-sample data also provide evidence of coding, even though, contrary to expectation, these hues were not given a common "red/blue" code. Instead, there was a significant tendency for the pigeons to code red as green, and blue as yellow. These data argue against the notion that the present coding effects are due solely to the use of natural color categories, because the red sample and blue sample coding effects clearly cut across the natural color categories found by Wright and Cumming (1971). Furthermore, if one assumes that coding reflects judgments of relative psychological similarity, the red-sample and blue-sample data suggest that the color spectrum for the pigeon may be psychologically linear (i.e., red is psychologically further from blue than it is from green) rather than circular as it is for humans (but see Schneider, 1972). At least the data suggest that for the pigeon the psychological distance between our red (peak transmission at $620 \mathrm{~nm}$ ) and our blue (peak transmission at $490 \mathrm{~nm}$ ) is greater than the psychological distance between our red and green and between our blue and yellow.

Although the coding effects were the only effects shown with all four sample colors, a significant new-correct/newincorrect comparison effect was found on both red-sample and blue-sample trials. On red-sample trials and bluesample trials, performance was better when the incorrect comparison was replaced than when the correct sample was replaced $(93.0 \%$ correct and $69.5 \%$ correct, respectively, for the red-sample trials and $85.8 \%$ correct and $69.1 \%$ correct, respectively, for the blue-sample trials). These data appear to support Cumming et al.'s (1965) conclusion that pigeons acquire conditional discriminations by learning an association between the sample and the correct comparison. But the fact that performance remains relatively high when the correct comparison is replaced (almost $70 \%$ correct) suggests that considerable learning about the incorrect comparison occurs as well. Furthermore, a reliable new-correct/new-incorrect comparison effect was not found for either yellow-sample or greensample trials. Yellow and green were the two sample colors for which very large coding effects were found. It may be that coding effects tend to obscure simpler samplespecific-association effects.

Group effects were found for two sample colors, yellow and blue, but the effects were not the same for the two colors. The group effect on yellow-sample trials showed up as a "concept" effect, or same-task effect; that is, Groups $\mathrm{MM}$ and $\mathrm{OO}$ performed better $(81.1 \%$ correct) than Groups $\mathrm{MO}$ and $\mathrm{OM}(73.6 \%$ correct). On bluesample trials, however, performance was better for the 
two groups for which the blue sample was part of a matching task (Groups MM and OM, $82.6 \%$ correct) than for the two groups for which the blue sample was part of an oddity task (Groups MO and OO, $72.3 \%$ correct). The superiority of matching over oddity performance is hard to interpret because (1) the effect was not found with the other colors, (2) a similar effect was not found in acquisition, and (3) matching superiority is not typically found when both tasks involve the same number of samplecomparison associations (Zentall \& Hogan, 1978).

Group $\times$ appropriateness interactions were found for two sample colors, yellow and red, but again the effects were not the same for the two colors. On red-sample trials, the interaction can best be described as the elimination of the appropriateness effect for Groups MM and OO, and on yellow-sample trials the interaction can best be described as a reduced effect of appropriateness for Group OO. Thus, coding appears to be reduced on redsample trials and to some extent on yellow-sample trials when the blue-green task is the same as the red-yellow task. These findings suggest a curious interaction between coding and identity concept learning. It may be that categorical coding and identity learning are to some extent mutually exclusive processes. Whatever conditions foster the development of one may tend to inhibit the development of the other.

Finally, a group $\times$ new-correct/new-incorrect interaction was found for red-sample trials. The superiority of new-incorrect trial performance over new-correct trial performance was found only when the red-sample trials were part of a matching task. Although this effect occurred only with red-sample trials, it is consistent with findings by Zentall et al. (1981) which show that, with matching tasks but not with oddity tasks, pigeons learn to associate the sample with the correct comparison stimulus. This matching/oddity asymmetry has been used to argue for the presence of an identity concept (see Zentall et al., 1981).

To summarize the major findings of the present experiment, in addition to confirming and extending findings of categorical color coding by pigeons (Wright \& Cumming, 1971; Zentall et al., 1981), the data also rule out failure to discriminate or confusion between hues as an explanation of the results. The data also raise questions about the shape of the pigeon's psychological hue dimension (i.e., is it linear or circular?). Finally, some of the data suggest that the relation between hue categorization and same/different concept learning may be inverse (i.e., conditions that encourage development of one may not encourage development of the other).

\section{REFERENCES}

Berlin, B., \& KAY, P. (1969). Basic color terms: Their universality and evolution. Berkeley: University of California Press.

Berryman, R., Cumming, W. W., Cohen, L. R., \& Johnson, D. F. (1965). Acquisition and transfer of simultaneous oddity. Psychological Reports, 17, 767-775.

BLough, D. S. (1957). Spectral sensitivity in the pigeon. Journal of the Optical Society of America, 47, 827-833.

Blough, D. S. (1959). Delayed matching in the pigeon. Journal of the Experimental Analysis of Behavior, 2, 151-160.

Boynton, R. M. (1972). Color vision. In J. W. Kling \& L. A. Riggs (Eds.), Woodworth and Schlosberg's Experimental psychology (Vol. 1). New York: Holt, Rinehart, and Winston.

Cumming, W. W., Berryman, R., \& Cohen, L. R. (1965). Acquisition and transfer of zero-delay matching. Psychological Reports, 17. 434-445.

HEIDER, E. R. (1972). Universals in color naming and memory. Journal of Experimental Psychology, 93, 10-20.

Lawrinck, D. H. (1963). The nature of a stimulus: Some relationships between learning and perception. In S. Koch (Ed.), Psychology: A study of a science (Vol. 5). New York: McGraw-Hill.

Prokasy, W. F., \& Hall, J. F. (1963). Primary stimulus generalization. Psychological Review, 70, 310-322.

ScInNEIDER, B. (1972). Multidimensional scaling of color difference in the pigeon. Perception \& Psychophysics, 12, 373-378.

SutuerlaAn, N. S., \& Mackintosh, N. J. (1971). Mechanisms of animal discrimination learning. New York: Academic Press.

Wright, A. A., \& Cumming, W. W. (1971). Color-naming functions for the pigeon. Journal of the Experimental Analysis of Behavior, 15, 7-17.

Zentall, T. R., Edwards, C. A. Möore, B. S., \& Hogan, D. E. (1981). Identity: The basis for both matching and oddity learning in pigeons. Journal of Experimental Psychology: Animal Behavior Processes, 7, 70-86.

Zentall, T. R., \& Hogan, D. E. (1974). Abstract concept learning in the pigeon. Journal of Experimental Psychology, 102, 393-398.

Zentall, T. R., \& Hogan, D. E. (1978). Same/different concept learning in the pigeon: The effect of negative instances and prior adaptation to transfer stimuli. Journal of the Experimental Analysis of Behavior, 30, 177-186.

Zentall, T. R., Hogan, D. E., Howard, M. M., \& Moore, B. S. (1978). Delayed matching in the pigeon: Effect on performance of sample-specific observing responses and differential delay behavior. Learning and Motivation, 9, 202-218.

(Manuscript received April 28, 1983; revision accepted for publication February 9, 1984.) 